\section{A CASE OF \\ CEREBRAL EMBOLISM FOURTEEN DAYS AFTER CONFINEMENT.}

\section{By ENGLEDUE PRIDEAUX, L.R. C.P. LOND., \&c.}

ELIZA S-, married, aged twenty-three, a primipara, was confined on Feb. 24th. There was nothing to call for remark in the confinement; it was a little tedious, but quite an ordinary one in every respect. She progressed favourably and did well, without any unusual symptom; and I ceased attending her at the usual time. On the fourteenth day after the confinement (March 10th) she had what her friends described as a "bilious attack," such as she had often had before. Her bowels had been constipated, for which she had taken castor oil, when she became sick, and vomited and retched very severely. Suddenly, according to the account of her friends, she complained of a violent pain on the right side of the temple which seemed to come on instantaneously. She was put to bed, and had a strong mustard poultice applied to the temple and became "very heavy in her head." The next day I saw her, and found her lying in bed apparently unconscious. After calling to her loudly, she awoke, showed me her tongue, recognised me, and spoke rationally; but in a few minutes relapsed into unconsciousness again. The right pupil was dilated and insensible to light; the left normal. Bowels constipated; heart sounds clear and regular; tongue foul and coated; pulse 70; temperature normal. Urine passed naturally; she had roused to pass it. I found it clear, with no albumen. The lochia had almost ceased; there was a very little discharge, slightly blood-stained. There was abundance of milk in the breasts, and she was awoke to suckle the child every now and then, and also to take her food; but she always relapsed into unconscionsness again. I gave her a dose of calomel and jalap, which moved the bowels several times, the motions being very dark.

The next day there was a slight improvement; she was more easily roused. She recognised her husband when he was sent for. Complained of my hand being cold. Pulse 70; temperature still normal; some slight paralysis of face on the right side; urine light coloured and turbid, full of phosphates, no albumen, and passed naturally. She took her food well; suckled the baby, but frequently relapsed into states of heavy unconsciousness. The afternoon of the same day her speech became muffled; she began to be more unconscious and difficult to rouse. The next day, March 14th, the fourth from the attack, she was quite unconscious, with stertorous breathing. Palse 100; temperature normal; urine passed in bed. After a great deal of rousing and shouting she put out her tongue, which was thickly coated; there was evident paralysis of the right arm. There was still milk in the breasts. I ordered her head to be shaved and applied a blister to the nape of the neck, and gave another powder of calomel and jalap. On the $15 \mathrm{th}$ there was still deepening coma. Urine passed in bed; pulse 100 ; temperature normal. There was still milk in the breasts ; in the afternoon she had two severe convulsions. She could swallow milk when put with a spoon into her mouth. On the l6th she had violent convulsions all night; the arm which was paralysed moved in the convulsions; both pupils dilated, the left slightly sensitive to a bright light. Pulse 120 ; temperature $102^{\circ}$. In the afternoon of the same day the breasts were dry, the patient being in a deep coma, severe convulsions constantly recurring; quite unable to swallow; died the same night.

Remarks. - This is a very unusual and rare termination of a puerperal case. I think there can be no doubt as to its being a case of embolism, the symptoms would indicate either that or apoplexy. The age of the patient would negative the latter. There was no heart disease, it is true, so that the formation of the embolus must have been due to the peculiar plastic condition of the blood in puerperal women, tending to the formation of clots, from one of which a portion might have been detached by plugging a cerebral artery on the right side of the cerebrum, or the clot might have been formed there in situ. It may have been that the straining and exertion caused by the vomiting and retching produced the detachment of the embolus; but I was inclined to think, although I was unable to get satisfactory information, that the sudden plugging of the vessel might have been, on the other hand, the cause of the attack of vomiting.
The apparent improvement that took place appears to have been due to partial recovery from the shock and to some reestablishment of the circulation, while the subsequent relapse and increase of the symptoms would be due to the more complete plugging of the vessel by an inflammatory condition, attended with exudation around the plug. That a case such as this is of rare occurrence is evidenced by the fact that I cannot find the subject mentioned in the ordinary text-books of midwifery, and in Playfair's work he quotes from a foreign author upon the subject, whilst it would appear that his few remarks are not derived from personal observation of a case.

Wellington, Somerset.

\section{A CASE OF BROMIDE RASH.}

BY PERCY BOULTON, M.D., M.R.C.P. LOND., PHYSICIAN TO THE SAMARITAN HOSPITAL FOR WOMEN AND CHILDREN AND

\section{GEORGE THIN, M.D.}

REMARKS BY DR. PERCY BOULTON.

MADAME V. G-, aged twenty-five, married, came to me at the out-patient department of the Samaritan Hospital early in October suffering from severe pelvic pain. I examined her uterine organs very carefully, and further than slight retroversion and endometritis there was nothing abnormal. The history was that six years ago she had a similar attack in Paris, and was in hospital there for six months before she was at all relieved. There was distinct periodicity about the pain, as it recurred each day at 4 P.M., and lasted about four hours.

My diagnosis was pelvic neuralgia in an exceedingly hysterical subject. I gave an injection of half $\mathbf{a}$ drachm of tincture of opium by the bowel, which speedily relieved her at the time. The pain returned, however, daily, and gradually got worse up to Oct. 12th, when I took her into hospital. Tincture of opium, administered as before, relieved her again, and I made an attempt to check the attacks, returning at four, by giving five grains of quinine in sweetened milk at 3.30 P.M.

From Oct. 12th to Oct. 19th I gave her three grains of bromide of iron three times a day in pill, when a few spots appeared about the face suggestive of a bromide rash, so I discontinued bromide altogether, About one drachm of bromide of iron had been taken altogether. I then gave the following pill three times a day : Perchloride of mercury, one-twenty-fourth of a grain ; sulphate of quinine, one grain; extract of belladonna, one-sixth of a grain; extract of hyos. cyamus, three grains. I had the uterus leeched, and applied pure carbolic acid to the cavity on several occasions.

On Oct. 25th the patient was much better, and anxious to return home. The attacks of pain had not recurred for some few days at 4 P.M. as before, but there was still at other times slight pelvic discomfort. The bowels were never in any way loaded, and the urine was examined and found normal, and the heart healthy.

On Nov. Ist Madame G - returned to me at the outpatient department, still better as regards her pelvic pain, but with her legs covered pretty uniformly with a discrete pustular eruption.

I was pretty certain from my previous examinations of her generative organs that it could not be specific, and, remembering that in the middle of October I had stopped the bromide of iron on account of a rash appearing, I had my suspicion of a bromide rash. As I was not sure on this point, and it was a remarkable case in its way, so very well marked, and yet so unlike anything $I$ had seen except pustular eczema (ecthyma), I asked Dr. Thin to see Madame $\mathrm{G}-$ and favour me with a diagnosis. He did so, and came to the conclusion that it was a bromide rash. The case was also seen at my clinique by Dr. Day, Dr. Reid, Mr. Meredith, and Mr. Stirling, and most of them were in favour of its being a specific rash.

\section{REMARKS BY DR. THIN.}

The chief interest in this case, in so far as the eruption is concerned, lies in the fact that, although the diagnosis was at the first glance by no means easy, it was yet possible to make out the nature of the rash from its objective characters alone. I diagnosed a bromide or iodide eruption before I was informed 
that either of these agents had been given. When the woman presented herself to me on November 2 nd, the anterior surfaces of both thighs and both legs were thickly studded with papules and pustules. There were a few on the back and on the shoulders and arms, but none on the rest of the body. Practically the seat of the eruption was the unusual one of the anterior surfaces exclusively of the lower extremities. The papules, I was informed, were the early stage of the pustules, the latter attaining the size of a large pea. The first appreciable stage was a small hard subcutaneous swelling.

The three stages of the lesion were thus induration, inflammation, and suppuration of a given point in the skin. The special character of the eruption was found in the last of these stages. The free bullous pustule in which the inflammation terminated is rarely found in any recognised form of skin disease. The lesions in certain forms of secondary syphilis most resembled it, but to my mind they were exclurled by the uncomplicated character of the pustule and by the localisation. A syphilitic dermatitis which produced so much free superficial pustulation would have been universal, and would have led either to an abundant formation of rupial crusts or to superficial ulceration.

But whilst the eruption differed from all known forms of skin disease it coincided in its principal characters with the lesions now known to be characteristic of certain forms of iodide and bromide eruptions.

Further inquiry elicited the information which is contained in Dr. Boulton's report in regard to the administration of the bromide of iron. The patient further informed me that the eruption on the legs began whilst she was taking the medicine, and that new papules had continued to appear up to the time I first saw her. The eruption was left to itself, and when I saw her ten days afterwards the smaller lesions had disappeared, and the position of the larger pustules was marked by dark-brown or copper-coloured spots, with dry adherent scales on the centre.

\section{INVERSION OF UTERUS; IMPENDING DEATH FROM SHOCK AND HÆMORRHAGE; FARADISATION ; RECOVERY.}

\section{BY R. H. BAKEWELL, M.D., \&C.}

ON May 11th, 1881, I was called in consultation to a primipara, aged about twenty-one, who had been attended by a midwife. After the birth of the child the midwife, finding something wrong, sent for Mr. D. P. James. He, on discovering the placenta in the bed and the uterus inverted, after an unsuccessful effort to reduce the inversion, sent for me. It was a little past 2 P.M. when I arrived, and found the patient exsanguine, pulseless, with cold extremities. On examination per vaginam the uterus was found inverted. Mr. James stated that when he tried to reduce the inversion the uterine action was so powerful that he could not succeed. I proposed that chloroform should be given, notwithstanding the very low condition of the patient, as it was obvious she must die from shock and hæmorrhage unless reduction were promptly effected. Chloroform was then administered by me, while Mr. James again attempted reduction. His hand became cramped, and he requested me to try what I could do. By placing the forefinger of my right hand in the left cornu of the uterus, and pushing it before me, at the same time grasping the uterine tumour through the abdominal parietes with the left hand, reduction was quickly effected. As soon as the inversion was reduced it was found that the uterus did not contract sufficiently, so, keeping my right hand in the cavity of the uterus, I directed the midwife to pour cold water on the abdominal walls. This caused firm contraction. There was still some bleeding from the cervix, so I placed a small plug saturated with solution of perchloride of iron in it. This completely stopped any further hæmorrhage. The patient was still in a very feeble state, and showed no signs of rallying. Brandy was freely administered, and beef-tea given. At half-past three I was obliged to leave. At 5 P.M. I returned, and found the patient much worse, although stimulants had been given in the interval. No pulse could be felt at the wrists, and the brachial pulse was very feeble; the respiration was of the Cheyne-Stokes character, the pause lasting from fifty-five to sixty seconds the whole body, and particularly the extremities, were cold, the lips livid, and the finger-nails purple. It being evident that death was imminent, and that shock was the chief cause of this state, as there had been no further hæmorrhage, I proposed faradisation. A masneto-electric battery was procured in a few minutes, and arplied along the course of the pneumogastric nerves and through the body by the hands. The index, which was divided into sixty degrees, was placed at fifteen, but no effect being produced in a few minutes it was advanced to twenty. Then the patient soon complained of "pins and needles," and shortly afterwards I was obliged to lessen the force of the current; the pulse became perceptible, the intervals of the Cheyne-Stokes respiration diminished by about five seconds each time until the breathing became regular; the body (which had been supplied with artificial heat) became warmer, and after faradisation had been administered for about an hour the patient was so much improved as to render its further use needless.

It was curious to note that as the patient revived under the faradisation the brandy, which had previously not acted at all, began to produce its usual effect; she began to talk nonsense, to laugh, and, though fully conscious of her condition, was evidently in the first stage of alooholic intoxication. At 9 P.M. the pulse was good, she had taken food, and was warm and comfortable.

On the fourth day after the labour the plug came away, and her recovery was uninterrupted by any bad symptom. Five weeks after the labour I made an examination both digital and specular, and found only a slight mucous discharge from the cervix, which, as well as the whole body of the uterus, was of normal size. There was a slight tenderness on pressure upwards between the uterus and bladder.

Hokitika, New Zealand.

\section{A tithirnor}

\section{HOSPITAL PRACTICE, BRITISH AND FOREIGN.}

Nulla autem est alia pro certo noscendi via, nisi quamplurimas et morborum et dissectionum historias, tum aliorum tum proprias collectas habere, et inter se comparare.-MongagNi De Sed. et Caus. Morb., lib. iv. Proœmium.

\section{LON DON HOSPITAL.}

CASE OF HYDROPHOBIA.

(Under the care of Dr. FraNCIS WARNER.)

THE attention of the public having been directed to the subjoined case, we are glad to be able to publish an accurate and authentic record. Though somewhat obscure in its nature and origin the diagnosis of hydrophobia is fairly made out. A comparison of this case with the one of traumautic tetanus recorded from the Wolverhampton Hospital will bring out more clearly many of the elements for forming a differential diagnosis between hydrophobia and tetanus. Fuller comments on these cases will be found in another column.

A young man aged nineteen, a starch worker, well nourished, with dark hair and eyes, was admitted at 1.30 P.M. Sept. 21st. When seen in bed he was the subject of violent and frequently recurring spasmodic paroxysms, bathed in clammy sweat, with the face and ears somewhat dusky. His head felt hot, and the temperature in the axilla was $101.8^{\circ} \mathrm{F}$. He was in a nearly unconscious condition and did not speak except once to ask for a handker. chief, and occasionally he swore. He sometimes smiled in a vague and meaningless manner. When a friend came to him he immediately looked at and appeared to recognise him, and seemed to attempt to articulate. No words, how. ever, could be distinguished. He was somewhat unmanageable, directed a kick with tolerable accuracy at the nurse, and threw a vessel at her, often spitting on those around him. During a paroxysm of spasm the lips were tightly closed, the head and neck were retracted, a noisy respi. ratory effurt then drove blood-stained saliva from the mouth, which was spat on to all objects around him; the jaws were closed by trismus. This spasm caused great cyanosis, the 